\title{
Why should we compare Wolf-Rayet codes?
}

\author{
Orsola De Marco ${ }^{1}$, Werner Schmutz ${ }^{1}$, Lars Koesterke ${ }^{2}$, Wolf-Rainer \\ Hamann $^{2}$, and Alex de Koter ${ }^{3}$ \\ ${ }^{1}$ Institute of Astronomy, Federal Institute of Technology, \\ Zürich, Switzerland \\ ${ }^{2}$ Department of Physics, University of Potsdam, Germany \\ ${ }^{3}$ Astronomical Institute Anton Pannekoek, University of Amsterdam, \\ the Netherlands
}

\begin{abstract}
We have modeled the spectrum of WR 135 with a co-moving frame code and then used identical input parameters with a Sobolev-approximation code. Helium lines compare well in strength and shape, while carbon lines are found to be much stronger in the co-moving frame code. The reason for this discrepancy is being investigated. The present comparison shows that tests have to be extended to more complex atomic models and compatibility between different codes should not be taken yet for granted.
\end{abstract}

\section{Comparison of the Kiel and the Sobolev-approximation codes}

In the co-moving frame Kiel-code (K, Hamann \& Schmutz 1988) photons interact with lines that have a finite width, where the continuum varies over a range of depth and frequency points. In the Sobolev-code (S, de Koter et al. 1993, 1997) the continuum opacity and source function as well as the level populations are considered constant within the line resonant volume. This approximation makes the $\mathrm{S}$ code much faster.

Test calculations have shown that the Sobolev-approximation is sufficiently accurate for O-and WR-type stars, while for LBVs adjustments in the mass-loss are necessary (de Koter et al. 1993). However, comparisons have so far been limited to atomic models comprising only hydrogen and helium. Here we include carbon in the model atom.

We have run a comparison based on the WC8 star WR 135. In Table 1, the parameters used as input to both models are listed. In Figure 1 we present line fits of helium and carbon and we compare physical quantities as a function of ionic density throughout the wind. Helium lines compare well, from which we conclude that the S-approximation holds for WR winds in the parameter space of this star. However C III-IV lines are predicted stronger by the K-code. The reason for this might be a different splitting of the super-levels or the lack of C III levels with $n>5$ in the S-code (Table 1; although the auto-ionizing levels present in both codes - should allow equal recombination rates). We have calculated an S-code model with $\mathrm{C} / \mathrm{He}=0.7$ to increase the carbon-line strengths, but with a larger $\mathrm{C} / \mathrm{He}$ ratio. The $\mathrm{He}$ I and $\mathrm{He}$ II lines cannot be matched simultaneously. Therefore the discrepancy cannot be removed by an adjustment of the model parameters. This comparison implies that the Sobolev-approximation might be responsible for the discrepancy. Until we can identify the exact cause 

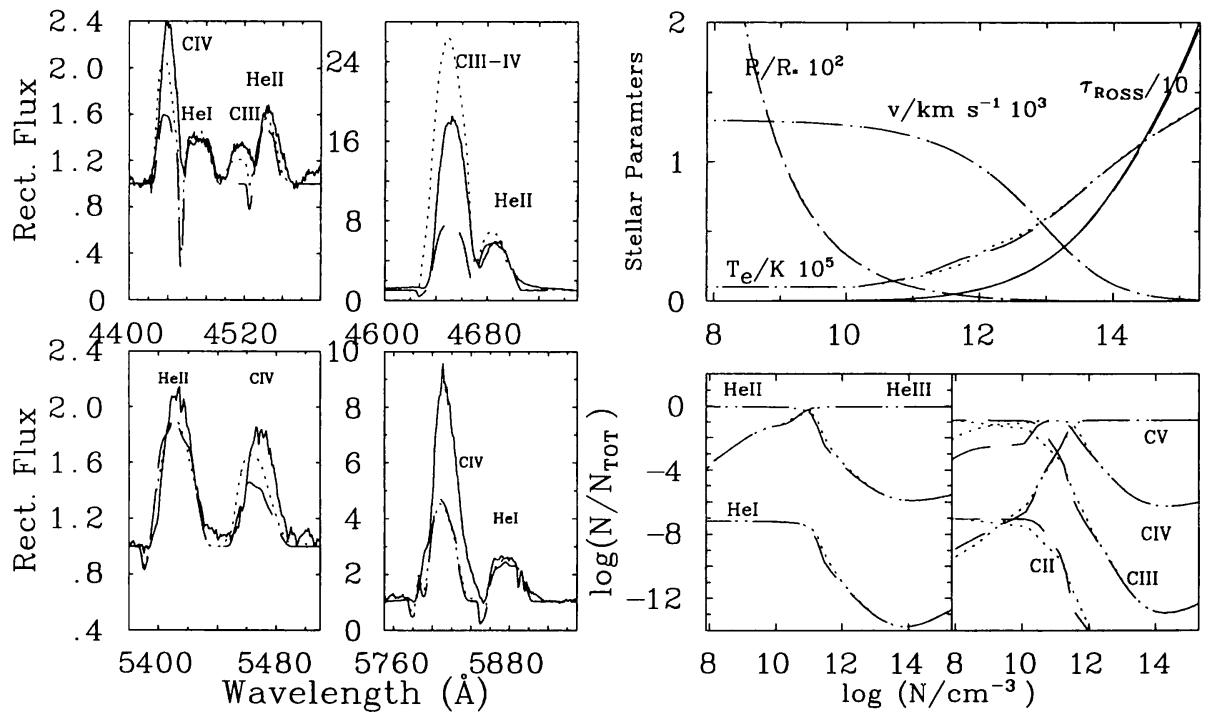

Figure 1. Line fits, stellar parameters and populations. Dotted line for the S-code, dashed for the K-code, solid for WR 135.

of the problem, we conclude that the S-code should not be used in the analysis of carbon lines.

Table 1. Input parameters (left) and atomic models (right). The K-code includes levels of C III with $n=5-7$ as super-levels; the S-code has individual levels only up to $n=5$. Both codes include levels of C IV up to $n=10$, where $n=6-10$ are super-levels; the S-code also groups all the C IV levels with $n=5$ into one super-level.

\begin{tabular}{lc|lcc}
\hline & & \multirow{3}{*}{} \\
$T_{\text {eff }}(\mathrm{K})$ & 75900 & ion & \multicolumn{2}{c}{ \# of levels } \\
$\mathrm{C} / \mathrm{He}($ by number $)$ & 0.1 & & K-code & S-code \\
$R_{\text {in }}\left(\mathrm{R}_{\odot}\right)$ & 2.6 & He I & 17 & 17 \\
$R_{\text {out }}\left(R_{*}\right)$ & 1000 & He II & 20 & 20 \\
$\log \left(L / \mathrm{L}_{\odot}\right)$ & 5.01 & He III & 1 & 1 \\
$\log \left(M / \mathrm{M}_{\odot} \mathrm{yr}^{-1}\right)$ & -4.1 & C II & 3 & 7 \\
$\mathrm{v}_{\infty}\left(\mathrm{km} \mathrm{s}^{-1}\right)$ & 1300 & C III & 40 & 44 \\
$\tau_{\text {Ross }}($ in $)$ & 20 & C IIIa-i & 31 & 31 \\
$v_{\text {Dop }}\left(\mathrm{km} \mathrm{s}^{-1}\right)$ & $100^{a}$ & C IV & 19 & 15 \\
no. of points & 89 & C V & 1 & 1 \\
& & & & \\
\hline
\end{tabular}

\section{References}

de Koter, A., Schmutz, W., Lamers, H. 1993, A\&A 277, 561

de Koter, A., Heap, S.R., Hubeny, I. 1997, ApJ 477, 792

Hamann, W.-R., Schmutz, W., Wessolowski, U. 1988, A\&A 194, 190 